\title{
Collisional line profiles of rubidium and cesium perturbed by helium and molecular hydrogen
}

\author{
N. F. Allard ${ }^{1,2}$ and F. Spiegelman ${ }^{3}$ \\ 1 Institut d'Astrophysique de Paris, UMR 7095, CNRS, Université Pierre et Marie Curie, 98bis boulevard Arago, 75014 Paris, France \\ e-mail: allard@iap.fr \\ 2 Observatoire de Paris-Meudon, LERMA, 92195 Meudon Principal Cedex, France \\ ${ }^{3}$ Laboratoire de Physique Quantique, UMR 5626, CNRS, Université Paul Sabatier, 118 route de Narbonne, 31400 Toulouse, France
}

Received 7 November 2005 / Accepted 29 December 2005

\section{ABSTRACT}

\begin{abstract}
Previous calculations of the far wings of the potassium and sodium doublets in a unified-line shape semi-classical theory have been used recently to improve theoretical modeling of synthetic spectra of brown dwarfs. However the comparison with observations shows missing opacity in the 0.85 to $1.1 \mu \mathrm{m}$ range. Contributions of other alkalis such as $\mathrm{Rb}$ I and Cs I may resolve this problem. We present new theoretical calculations of the line profiles of these two alkalis perturbed by helium and molecular hydrogen.
\end{abstract}

Key words. line: profiles - radiation mechanisms: general - stars: atmospheres - stars: low-mass, brown dwarfs

\section{Introduction}

The construction of model atmospheres and synthetic spectra for cool brown dwarfs and extrasolar giant planets is necessary to derive reliable atmospheric parameters and the surface chemical composition for these objects. The resonance lines of alkali elements can form in deep regions of the atmosphere, and are the rare remaining sensitive spectral type and atmospheric parameter indicators. The importance of the far wings of the potassium doublet, centered at $0.77 \mu \mathrm{m}$ in the spectra of methane brown dwarfs, has been demonstrated by Burrows et al. (2000). Alkali line profiles were calculated by Burrows \& Volobuyev (2003) using multiconfiguration self-consistent field Hartree-Fock potentials in the Szudy \& Baylis $(1975,1996)$ theory. In Allard et al. (2003) we presented the first application of the absorption profiles of sodium and potassium perturbed by helium and molecular hydrogen to the modeling of brown dwarfs.

Line profile calculations have been done in a classical path theory that takes into account the variation of the electric dipole moment during a collision (see Allard et al. 1999). Our approach requires prior knowledge of accurate theoretical molecular potentials to describe the interaction between radiator and perturber, and also knowledge of the variation in the radiative dipole moment with atom-atom separation for each molecular state. The calculations were based on the molecular potentials of Pascale (1983) for Na/K-He and of Rossi \& Pascale (1985) for $\mathrm{Na} / \mathrm{K}-\mathrm{H}_{2}$.

The new line profiles were included as a source of opacity in model atmosheres and synthetic spectra using the Allard et al. (2001) atmosphere program PHOENIX. The results were compared to previous models (Allard et al. 2003), and demonstrated that these improvements are of fundamental importance for obtaining a better quantitative interpretation of the spectra. The new line profiles provide increased opacities in the optical spectra, although the potassium and sodium far wings alone cannot explain the missing opacity in the 0.85 to $1.1 \mu \mathrm{m}$ range. The contribution of rubidium and cesium, described with Lorentz profiles using van der Waals damping constants, may be the cause of the remaining discrepancy. In this paper, we present the first calculation of accurate line profiles of rubidium and cesium perturbed by $\mathrm{He}$ and $\mathrm{H}_{2}$. The potential curves used in the calculations are those given by Pascale (1983) and Rossi \& Pascale (1985) to describe the interactions of alkalis first with $\mathrm{He}$ and later with $\mathrm{H}_{2}$. For the specific application to heavy alkalis, we need to take the spin-orbit coupling of the alkali into account. This is done using an atom-in-molecule intermediate spin-orbit coupling scheme, analogous to the one derived by Cohen \& Schneider (1974). The degeneracy is partially split by the coupling and the distinction between $D 1\left(P_{1 / 2}\right)$ and $D 2$ $\left(P_{3 / 2}\right)$ results. The previous work on the light alkalis presented in Allard et al. $(2003,2005)$ used Hund's case $a$ or $b$ for determining the shape of spectral line and neglected the fine structure that was insignificant in the far wing.

In Sect. 2 we recall the dipole autocorrelation formulation of the atomic line shape in a classical path trajectory approximation. In Sects. 3 and 4 we describe the calculations of the potentials and the scheme used to account for spin-orbit coupling. The calculation of dipole moments for fine structure states is also described. In Sect. 5 we present $\mathrm{Rb}$ and $\mathrm{Cs}-\mathrm{He} / \mathrm{H}_{2}$ profiles for the conditions encountered in the atmosphere of brown dwarfs and extra-solar giant planets.

\section{General expression for the spectrum}

We will be concerned with the pressure broadening by neutral foreign gas of an isolated spectral line at densities of the foreign gas extending well above the binary collision impact region. Following the work of Baranger (1958a,b), we derived a classical path expression for a pressure-broadened spectral line shape allowing for degenerate atomic states and radiative dipole moments that vary with $R(t)$, the internuclear distances (Allard et al. 1999). 
We here consider an optically active atom, the radiator, surrounded by moving perturbers. The power radiated in a dipole transition $\alpha=(i, f)$ from initial state $i$ to final state $f$ is given by

$P_{\alpha}(\Delta \omega)=\frac{4 \omega_{i f}^{4}}{3 c^{3}} \frac{N_{i}}{g_{i}} \sum|\langle f|\boldsymbol{d}| i\rangle|^{2}$,

where $\omega_{i f}$ is the Bohr angular frequency

$\omega_{\alpha} \equiv \omega_{f i} \equiv\left(E_{f}^{\infty}-E_{i}^{\infty}\right) / \hbar$,

$N_{i}$ the population of the initial level, $g_{i}$ the statistical weight, $c$ the velocity of the light, and $\boldsymbol{d}$ the dipole moment of the radiator. The summation $\sum$ must be extended over all the degenerate substates of the system.

Dropping the factor $\frac{4 \omega_{i f}^{4}}{3 c^{3}} N_{i}$ in the above expression, we get the distribution $I(\omega)$ commonly referred to as the spectrum or line shape. This spectrum $I(\Delta \omega)$ can be written as the Fourier transform (FT) of the dipole autocorrelation function $\Phi(\mathrm{s})$,

$I(\Delta \omega)=\frac{1}{\pi} \operatorname{Re} \int_{0}^{+\infty} \Phi(s) \mathrm{e}^{-\mathrm{i} \Delta \omega s} \mathrm{~d} s$.

The autocorrelation function $\Phi(s)$ is calculated with the assumptions that the radiator is stationary in space, the perturbers are mutually independent, and in the adiabatic approach the interaction potentials give contributions that are scalarly additive. This last simplifying assumption allows us to calculate the total profile $I(\omega)$, when all the perturbers interact, as the FT of the $N$ th power of the autocorrelation function $\phi(s)$ of a unique atomperturber pair. Therefore

$\Phi(s)=(\phi(s))^{N}$,

that is to say, we neglect the interperturber correlations. The radiator can interact with several perturbers simultaneously, but the perturbers do not interact with each other. It is what Royer (1980) calls the totally uncorrelated perturbers approximation. The fundamental result expressing the autocorrelation function for many perturbers in terms of a single perturber quantity $g(s)$ was first obtained by Anderson \& Talman (1956) and Baranger (1958a) in the classical and quantum cases, respectively.

We obtain for a perturber density $n_{\mathrm{p}}$

$\Phi(s)=\mathrm{e}^{-n_{\mathrm{p}} g(s)}$,

where decay of the autocorrelation function with time leads to atomic line broadening. For a transition $\alpha=(i, f)$ from initial state $i$ to final state $f$, we have

$$
\begin{aligned}
g_{\alpha}(s) & =\frac{1}{\sum_{e, e^{\prime}}(\alpha)\left|d_{e e^{\prime}}\right|^{2}} \sum_{e, e^{\prime}}{ }^{(\alpha)} \\
& \int_{0}^{+\infty} 2 \pi \rho \mathrm{d} \rho \int_{-\infty}^{+\infty} \mathrm{d} x \tilde{d}_{e e^{\prime}}[R(0)] \\
& \times\left[\mathrm{e}^{\frac{\mathrm{i}}{\hbar} \int_{0}^{s} \mathrm{~d} t V_{e^{\prime} e}[R(t)]} \tilde{d}_{e e^{\prime}}^{*}[R(s)]-\tilde{d}_{e e^{\prime}}[R(0)]\right] .
\end{aligned}
$$

We define $\tilde{d}_{e e^{\prime}}(R(t))$ as a modulated dipole (Allard et al. 1999)

$$
D(R) \equiv \tilde{d}_{e e^{\prime}}[R(t)]=d_{e e^{\prime}}[R(t)] \mathrm{e}^{-\frac{\beta}{2} V_{e}[R(t)]},
$$

where $\beta$ is the inverse temperature $(1 / k T)$. Here $V_{e}$ is the ground state potential when we consider absorption profiles, or an excited state for the calculation of a profile in emission.

\section{Adiabatic molecular potentials}

We consider the system composed of a radiating alkali atom $A$ in collision with an atom or molecule $B$, separated from $A$ by distance $R$. In the case of an alkali- $\mathrm{H}_{2}$ collision, $B$ is situated at the center of mass of $\mathrm{H}_{2}$. In Eq. (6) $e$ and $e^{\prime}$ label the energy surfaces on which the interacting atoms approach the initial and final atomic states of the transition as $R \rightarrow \infty$. The $\operatorname{sum} \sum_{e, e^{\prime}}^{(\alpha)}$ is over all pairs $\left(e, e^{\prime}\right)$ such that $\omega_{e^{\prime}, e}(R) \rightarrow \omega_{\alpha}$ as $R \rightarrow \infty$.

In the present context, the perturbation of the frequency of the atomic transition during the collision results in a phase shift, $\eta(s)$, calculated along a classical path $R(t)$ assumed to be rectilinear, is

$\eta(s)=\frac{\mathrm{i}}{\hbar} \int_{0}^{s} \mathrm{~d} t V_{e^{\prime} e}[R(t)]$

where $\Delta V(R)$, the difference potential, is given by

$\Delta V(R) \equiv V_{e^{\prime} e}[R(t)]=V_{e^{\prime}}[R(t)]-V_{e}[R(t)]$,

and represents the difference between the electronic energies of the quasimolecular transition. The potential energy for a state $e$ is

$V_{e}[R(t)]=E_{e}[R(t)]-E_{e}^{\infty}$.

At time $t$ from the point of closest approach

$R(t)=\left[\rho^{2}+(x+\bar{v} t)^{2}\right]^{1 / 2}$

with $\rho$ the impact parameter of the perturber trajectory and $x$ the position of the perturber along its trajectory at time $t=0$.

\section{1. $\mathrm{Rb} / \mathrm{Cs}-\mathrm{He}$ diatomic potentials}

Molecular-structure calculations have been performed by Pascale (1983) to obtain the adiabatic potentials for ground states and numerous excited states of alkali-metal-He systems. They use $l$-dependent pseudopotentials defined from spectroscopy data or scattering data. Potential energy curves for the $\mathrm{Rb} / \mathrm{Cs}-\mathrm{He}$ molecules in the $A^{2} \Pi$ and $B^{2} \Sigma$ states are shown in Figs. 1 and 2 (dotted curves). As the spin-orbit interaction is not included, there are two electronic states connecting to the $\mathrm{Rb}(5 \mathrm{p}) / \mathrm{Cs}(6 \mathrm{p})+$ He limit.

\section{2. $\mathrm{Rb} / \mathrm{Cs}-\mathrm{H}_{2}$ diatomic potentials}

The alkali- $\mathrm{H}_{2}$ molecular potentials were evaluated by Rossi \& Pascale (1985) for the two symmetries $\mathrm{C}_{2 v}\left(\Theta=90^{\circ}\right)$ and $\mathrm{C}_{\infty v}$ $(\Theta=0)$, where $\Theta$ is the angle between the direction of the molecular axis and the internuclear axis. The molecule $\mathrm{H}_{2}$ is assumed to be in its ground state and its bond length fixed to the equilibrium value $r_{e}=1.4$ a.u. For $\Theta=90^{\circ}$, the ground state that we label $X$ is a state of ${ }^{2} A_{1}$ symmetry, and the excited states are of ${ }^{2} A_{1},{ }^{2} B_{1}$, and ${ }^{2} B_{2}$ symmetry.

The $R$ dependence of the electronic energies $V\left(R, r_{e}, \Theta\right)$ of the first excited states for $\Theta=0$ and $90^{\circ}$ are reproduced in Figs. 3 and 4 for the rubidium and Figs. 5 and 6 for the cesium (dotted curves).

The spin-orbit interaction is not included in their calculations. In the case of light alkalis the fine structure is small and the system may be described by Hund's case $a$ or $b$ in the molecular region, where the wing is formed. For heavy alkalis fine structure has to be included. 


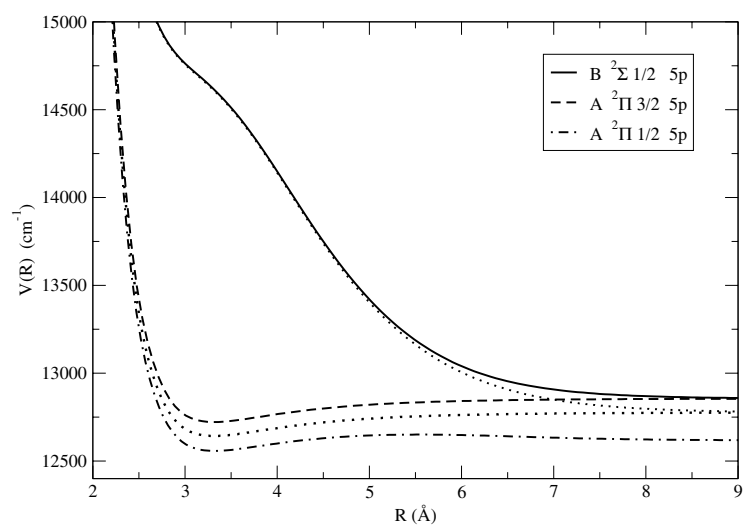

Fig. 1. Potential curves for the $A$ and $B$ states of the $\mathrm{Rb}-\mathrm{He}$ molecule with and without spin-orbit coupling (dotted curves).

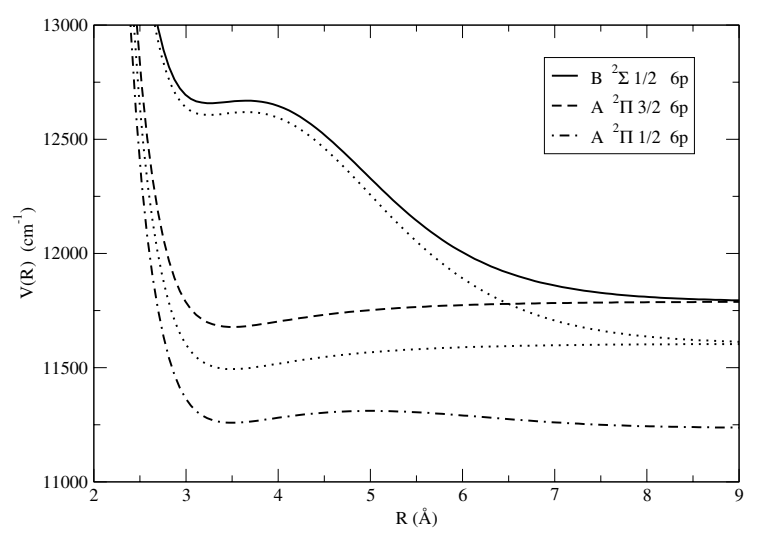

Fig. 2. Potential curves for the $A$ and $B$ states of the Cs-He molecule with and without spin-orbit coupling (dotted curves).

\subsection{SO coupling}

Spin-orbit coupling was included within the atom-in-moleculelike scheme introduced by Cohen \& Schneider (1974). This scheme relies on a monoelectronic formulation of the spin-orbit coupling operator

$H_{\mathrm{SO}}=\sum_{i} h_{\mathrm{SO}}(i)$.

The total Hamiltonian $H_{\mathrm{el}}+H_{\mathrm{SO}}$ is expressed in the basis set of the eigenstates of the purely electrostatic Hamiltonian $H_{\mathrm{el}}$. The spin-orbit coupling between these molecular many-electron doublet states $\Phi_{k \sigma}$ (approximated for the present purpose as determinants) is isomorphic to that between the open shell molecular spin-orbitals $\phi_{k \sigma}$, correlated with the six $p$ spin-orbitals of the alkali atom (We use here the Cartesian orbital representation, where $k=x, y, z$ labels the space part and $\sigma=\alpha, \beta$ labels the spin projection), so that

$\left\langle\Phi_{k \sigma}\left|h_{\mathrm{SO}}\right| \Phi_{l \tau}\right\rangle=\left\langle\phi_{k \sigma}\left|h_{\mathrm{SO}}\right| \phi_{l \tau}\right\rangle$.

The Cohen \& Schneider approximation consists of setting these matrix elements equal to their asymptotic (atomic) values

$\left\langle\phi_{k \sigma}(\boldsymbol{R})\left|h_{\mathrm{SO}}\right| \phi_{l \tau}(\boldsymbol{R})\right\rangle=\left\langle\phi_{k \sigma}(\infty)\left|h_{\mathrm{SO}}\right| \phi_{l \tau}(\infty)\right\rangle$.

This scheme makes no a priori assumption about the magnitude of spin-orbit coupling versus pure electrostatic interactions and allows general molecular intermediate coupling.

The effective single-electron spin-orbit operator is taken as

$h_{\mathrm{SO}}=\zeta \hat{l} . \hat{s}$

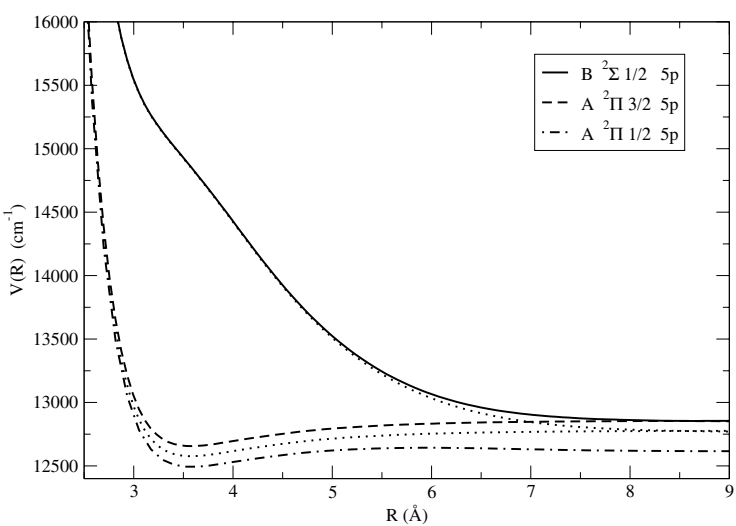

Fig. 3. $\mathrm{Rb}-\mathrm{H}_{2}$ potentials for the resonance lines for the $\mathrm{C}_{\infty v}$ symmetry with and without spin-orbit coupling (dotted curves).

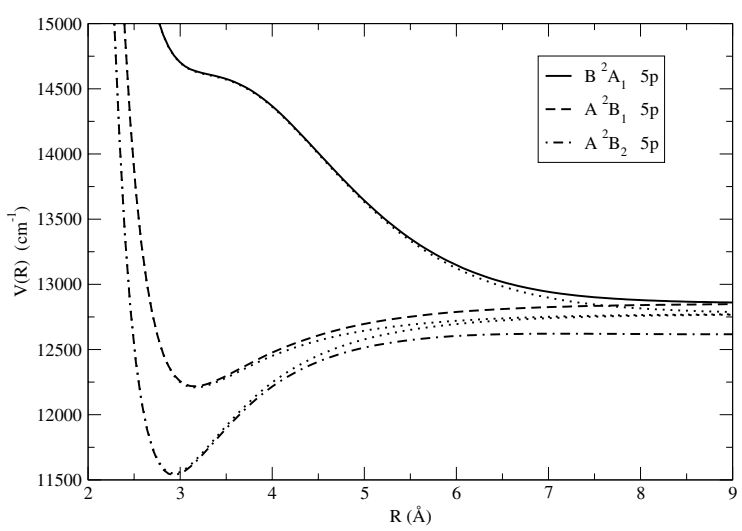

Fig. 4. $\mathrm{Rb}-\mathrm{H}_{2}$ potentials for the resonance lines for the $\mathrm{C}_{2 v}$ symmetry with and without spin-orbit coupling (dotted curves).

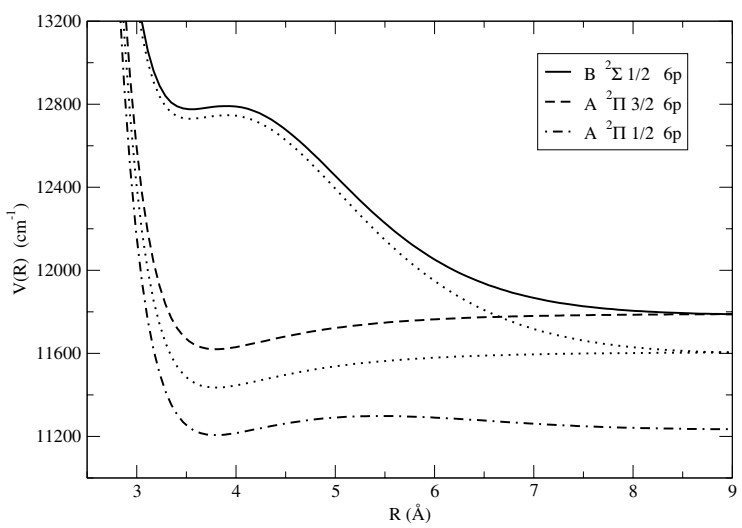

Fig. 5. $\mathrm{Cs}-\mathrm{H}_{2}$ potentials for the resonance lines for the $\mathrm{C}_{\infty v}$ symmetry with and without spin-orbit coupling (dotted curves).

with $3 \frac{\zeta}{2}=E\left({ }^{2} P_{\frac{3}{2}}\right)-E\left({ }^{2} P_{\frac{1}{2}}\right)$ the atomic spin-orbit constant for the alkali doublets: $\zeta_{5 p}=158 \mathrm{~cm}^{-1}$ for rubidium and $\zeta_{6 p}=$ $369 \mathrm{~cm}^{-1}$ for cesium.

The total Hamiltonian matrix $H=H_{\mathrm{el}}+H_{\mathrm{SO}}$ is a $6 \times 6 \mathrm{com}-$ plex matrix,

$\mathbf{H}_{\mathrm{el}}+\mathbf{H}_{\mathrm{SO}}=\left(\begin{array}{cccccc}E_{X} & 0 & -\mathrm{i} \frac{\zeta}{2} & 0 & 0 & \frac{\zeta}{2} \\ 0 & E_{X} & 0 & \mathrm{i} \frac{\zeta}{2} & \frac{\zeta}{2} & 0 \\ \mathrm{i} \frac{\zeta}{2} & 0 & E_{Y} & 0 & 0 & -\mathrm{i} \frac{\zeta}{2} \\ 0 & -\mathrm{i} \frac{\zeta}{2} & 0 & E_{Y} & -\frac{\zeta}{2} & 0 \\ .0 & \frac{\zeta}{2} & 0 & \mathrm{i} \frac{\zeta}{2} & E_{Z} & 0 \\ \frac{\zeta}{2} & 0 & \frac{\zeta}{2} & 0 & 0 & E_{Z}\end{array}\right)$. 


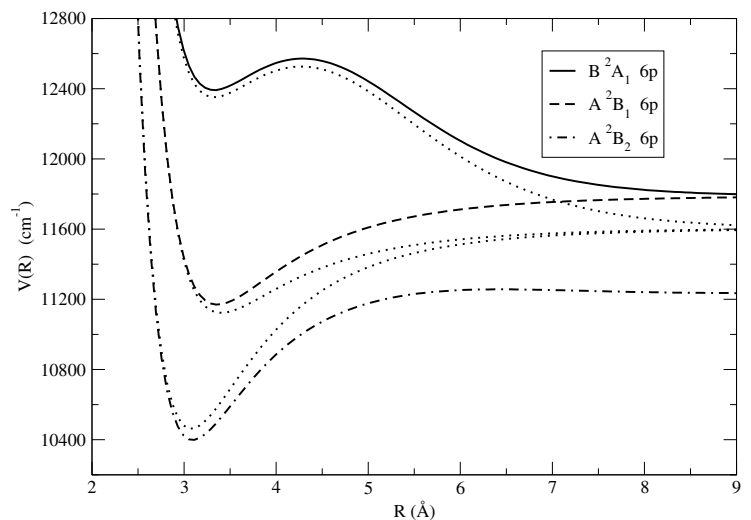

Fig. 6. Cs- $\mathrm{H}_{2}$ potentials for the resonance lines for the $\mathrm{C}_{2 v}$ symmetry with and without spin-orbit coupling (dotted curves).

For alkali- $\mathrm{H}_{2}$ in $C_{\infty v}$ geometry, or alkali-He, the electrostatic Hamiltonian is defined by setting $E_{X}=E_{Y}=E\left({ }^{2} \Pi\right)$ and $E_{Z}=$ $E\left({ }^{2} \Sigma\right)$ (considering the symmetry axis as the $z$ axis). In linear geometries, the matrix can be unitarily-transformed and blockdecomposed into smaller real matrices according to the values of the total angular momentum along the molecular axis $\Omega= \pm \frac{3}{2}$, $\pm \frac{1}{2}$. However, the formulation is not restricted to the linear case and takes into account the further degeneracy splitting occuring for alkali- $\mathrm{H}_{2}$ in the $C_{2 v}$ geometry, setting $E_{Z}=E\left({ }^{2} A_{1}\right)$, $E_{X}=E\left({ }^{2} B_{2}\right), E_{Y}=E\left({ }^{2} B_{1}\right)$. In any case, the diagonalization of the above matrix at each internuclear distance provides the spin-orbit coupled eigenstates

$\Psi_{e^{\prime}}=\sum_{k \sigma} c_{k \sigma}^{e^{\prime}} \Phi_{k \sigma}$

\section{Radiative dipole transition moments}

Since there is no spin-orbit coupling, the two ground-state components $e$ can be labelled according to the spin projection $\tau=$ $\alpha, \beta$ of their external electron. The dipole moment from these components $\Phi_{\tau}$ to the spin-orbit coupled upper states $\Psi_{e^{\prime}}$ were calculated from the coefficients of the latter on the spin-orbitless states $\Phi_{k \sigma}$

$D_{\tau e^{\prime}}(R)=\sum_{k \sigma} c_{k \sigma}^{e^{\prime}}(R) D_{\tau k \sigma} \delta_{\tau \sigma}(R)=\sum_{k} c_{k \tau}^{e^{\prime}}(R) D_{\tau k}(R)$

In the case of alkali-He, the spin-orbitless $D_{\tau k}(R)$ transition moments $\left(X^{2} \Sigma-B^{2} \Sigma\right)$ and $\left(X^{2} \Sigma-B^{2} \Pi\right)$ were provided by Pascale (2003). For the alkali- $\mathrm{H}_{2}$ systems, they were assumed to remain constant through out the collision and equal to their asymptotic value.

\section{Interpretation of the absorption profiles}

The theoretical absorption spectra of the light alkalis $\mathrm{Li}, \mathrm{Na}$, and $\mathrm{K}$ are reported in Allard et al. (2003, 2005). In these calculations, which neglected the fine structure, we used the Hund's case $b$ to determine the wings and the Hund's case $c$ to determine the line parameters of the center of the $D 1$ and $D 2$ lines. Using the interaction potentials described above allows us to make a distinction between the contribution of the $D 1$ and $D 2$ line wings.

\subsection{D2 lines}

Two excited molecular levels $B^{2} \Sigma_{1 / 2}$ and $A^{2} \Pi_{3 / 2}$ contribute to the $D_{2}$ line and produce opposite wings. While $A$ states radiate on the red wing, $B$ states radiate on the blue wing. One of the most striking qualitative features that often appears in a line wing is an intensity maximum called a satellite band. Extensive observations have been made on the line satellites appearing in absorption and emission on the principal-series line of the alkali metal pressurized by inert gases. The line satellites are induced not only by the inert gases but also by perturbers such as $\mathrm{H}_{2}$, $\mathrm{D}_{2}, \mathrm{~N}_{2}$, and many hydrocarbons (Allard \& Kielkopf 1982). Blue satellite bands are correlated with maxima in the excited $B$ state potentials and can be predicted from the maxima in the difference potentials $\Delta V$ for the $B-X$ transition.

\subsection{1. $\mathrm{Rb}-\mathrm{He} / \mathrm{H}_{2}$ collisional profiles}

The difference potential energy as shown in Fig. 7 gives rise to satellites in the far wings of the $D 2$ line positioned at $\Delta \omega=$ $\Delta V_{\max }$ (see e.g. Allard 1978; Royer 1978). We show in Fig. 9 the resulting profiles for the $D 2$ lines of the different pairs in collisions. To make the description of the profiles easier and to point out the relationship between the extrema of $\Delta V$ and the position of the satellites, we display the variation in the line profile with $\Delta \omega$, the frequency difference between the center of the unperturbed spectral line and the current $\omega$, measured for convenience in the same units as the potential energy difference. For the $\mathrm{Rb}$-He pair, $\Delta V(R)$ is maximum at an internuclear separation of $3.5 \AA$, and the maximum at $1110 \mathrm{~cm}^{-1}$ leads to a line satellite at $1010 \mathrm{~cm}^{-1}(0.73 \mu \mathrm{m})$. The position of the satellites is taken at the local maximum, whenever possible. When they only appear as a shoulder, their position is then evaluated approximately.

The perturber densities used in the calculations are those encountered in the atmospheres of brown dwarfs. At moderate pressures the line profile intensities are related to the perturbations caused by a single binary collision event. But the density of molecular hydrogen can reach $10^{20} \mathrm{~cm}^{-3}$, and for this density multiple perturber effects can appear in the blue wing of the $D 2$ line. If one assumes additive-pair interactions, a satellite feature at $\Delta V$ in the binary spectrum will also appear in the spectrum as a weaker satellite at $2 \Delta V$ due to the presence of a second perturber in the volume of close interaction. Such multiple satellites have been experimentally observed at very high densities (Mc Cartan \& Hindmarsh 1969; Kielkopf \& Allard 1979). This series of satellites corresponds to the simultaneous presence of $k$ perturbers in the collision volume $V_{\text {coll }}$. For $\mathrm{Rb}-\mathrm{He}$ we notice in Fig. 9 the first satellite at $1010 \mathrm{~cm}^{-1}(0.73 \mu \mathrm{m})$ and the second one at $1960 \mathrm{~cm}^{-1}(0.67 \mu \mathrm{m})$. The contribution of the $\mathrm{Rb}-\mathrm{H}_{2}$ for the two orientation angles of $\mathrm{H}_{2}$ gives satellites at 1300 and $1050 \mathrm{~cm}^{-1}$, which appear as two bumps at 0.7 and $0.72 \mu \mathrm{m}$ on the total profile (Fig. 11). Taking all the orientations into account should lead to a large band with an extension of $200 \AA$.

While the position of the line satellites critically depends on the interaction potential, their amplitude depends on both the interaction potential and the dipole moments. The satellite amplitude depends on the value of the electric dipole transition moment in the internuclear region where the line satellite is formed (Allard et al. 1998). The importance of the fast variations with $R$ of the exponential factor $\mathrm{e}^{-\frac{\beta}{2} V_{\mathrm{e}}[R(t)]}$ in $\tilde{d}_{e e^{\prime}}(R(t))$, the modulated dipole moment, can be seen in Fig. 8, where we displays $D(R)$ together with the corresponding $\Delta V(R)$ for the $\mathrm{Rb}-\mathrm{He}$ pair. The strength of the satellites increases with temperature, so this explains why the second satellite appears distinctly at $3000 \mathrm{~K}$ and 


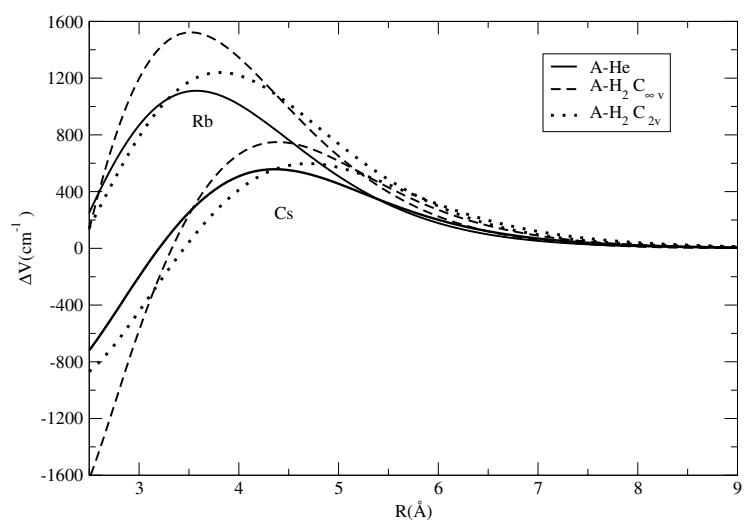

Fig. 7. Difference $\Delta V$ of upper and lower state potentials for the $B-X$ transition of $\mathrm{D} 2$ line of $\mathrm{Rb} / \mathrm{Cs}-\mathrm{He} / \mathrm{H}_{2}$ systems.

as a changing of slope on the blue wing at $1000 \mathrm{~K}$ (Figs. 9 and 13).

\subsubsection{Cs- $\mathrm{He} / \mathrm{H}_{2}$ collisional profiles}

The amplitude of the binary satellite is proportional to the perturber density, whereas the amplitude of the secondary one varies with the square of the perturber density; but for a given density, a larger position of the maximum in the difference potential leads to a larger number of perturbers in the close interaction volume. The feature grows as the number of perturbers in the region of satellite formation increases and consequently a larger amplitude of the satellite is expected (see e.g. Allard et al. 1994). The examination of Fig. 7 leads us to expect that satellites associated with the resonance line of cesium are more important than those associated with rubidium, as $\Delta V(R)$ is a maximum at an internuclear separation of $4.5 \AA$ instead of $3.5 \AA$ for rubidium. In Fig. 13 the second satellite appears as a shoulder on the $\mathrm{Cs}-\mathrm{He}$ profile at $1000 \mathrm{~K}$. Because of the closer proximity of the Cs satellite to the line $\left(\Delta V(R) \sim 600 \mathrm{~cm}^{-1}\right)$, the second satellite is smeared out in the increasing blue wing with temperature and remains a shoulder even at $3000 \mathrm{~K}$ (Fig. 10).

Gilbert \& Ch'en (1969) observed the Cs-He satellite and reported a separation of the satellite from the $D 2$ line equal to $387 \pm 9 \mathrm{~cm}^{-1}$, which agrees rather well with our calculation. Figure 10 shows a Cs-He satellite at $420 \mathrm{~cm}^{-1}$ that corresponds to $0.82 \mu \mathrm{m}$ (Fig. 13). The blue wings decrease abruptly far beyond the position of the line satellites.

Similar properties are obtained for the $\mathrm{Cs}-\mathrm{H}_{2}$ systems in collision. The maxima at 600 and $750 \mathrm{~cm}^{-1}$ in the difference potentials (Fig. 7) give rise to satellites at $495 \mathrm{~cm}^{-1}(0.82 \mu \mathrm{m})$ and $635 \mathrm{~cm}^{-1}(0.81 \mu \mathrm{m})$. In Fig. 12, the blue satellites of Cs- $\mathrm{H}_{2}$ appear from 0.81 to $0.82 \mu \mathrm{m}$.

\subsection{D1 lines}

The blue wings of the $D 1$ line decrease very rapidly due to the shape of the potential of the $A^{2} \Pi_{1 / 2}$ state, while the red wing extends until $2000 \AA$ from the line center (Figs. 11-13).

\subsection{Comparison with a Lorentzian}

A collisionally broadened line has a Lorentzian profile near the line center that can be related to the FT of a radiative wave in which short duration collisions produce sudden phase changes. In the theory of impact broadened line shape, the phase shifts are

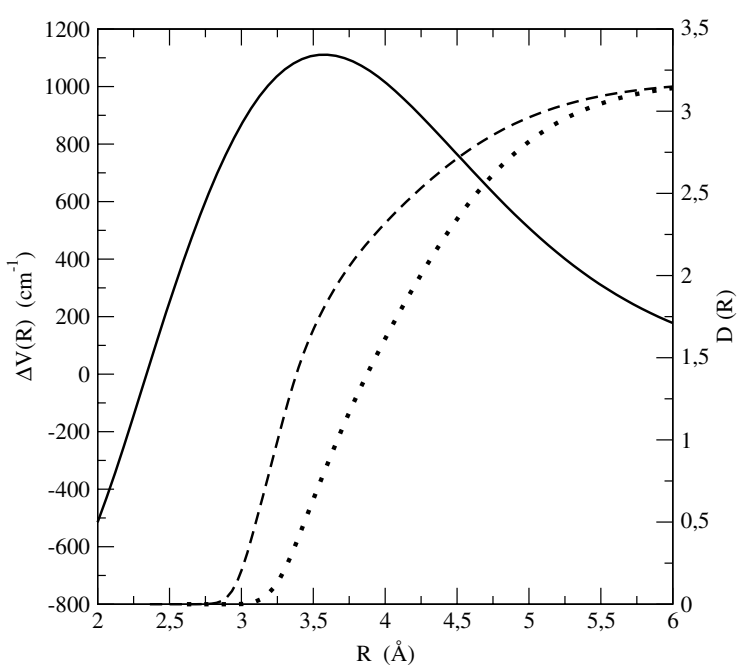

Fig. 8. $\Delta V$ and variation with temperatures of Rb-He modulated dipole at 3000 and $1000 \mathrm{~K}$ (top to bottom).

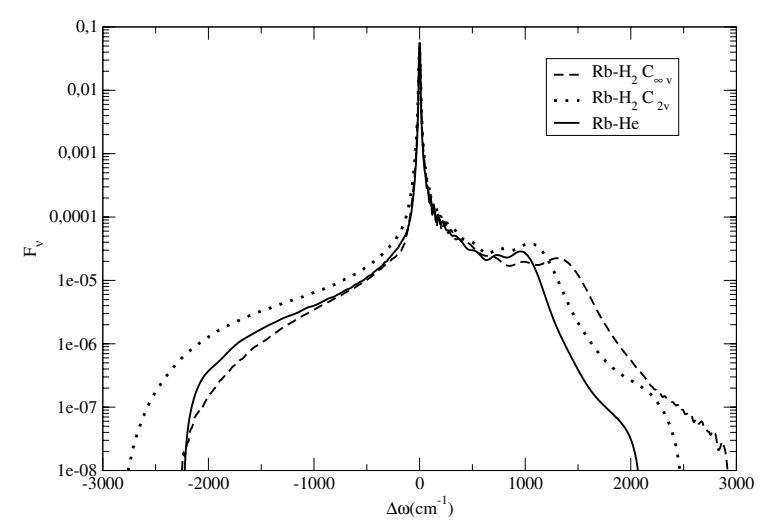

Fig. 9. $\mathrm{Rb}$ line profiles perturbed by $\mathrm{He}$ and by $\mathrm{H}_{2}$ for the $C_{2 v}$ and $C_{\infty v}$ symmetries. The density of perturbers is $n_{\mathrm{H}_{2}}=10^{20} \mathrm{~cm}^{-3}$. The temperature is $3000 \mathrm{~K}$.

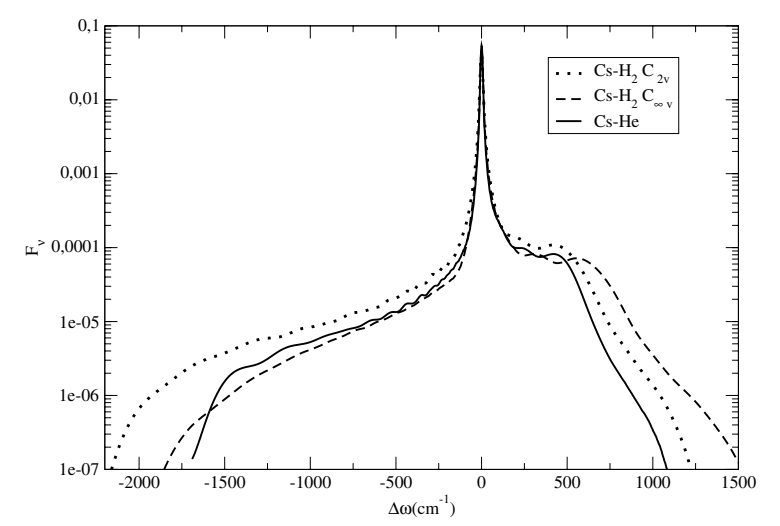

Fig. 10. Cs line profiles perturbed by $\mathrm{He}$ and by $\mathrm{H}_{2}$ for the $C_{2 v}$ and $C_{\infty v}$ symmetries. The density of perturbers is $n_{\mathrm{H}_{2}}=10^{20} \mathrm{~cm}^{-3}$. The temperature is $3000 \mathrm{~K}$.

given by Eq. (8) with the integral taken between $s=0$ and $\infty$. At sufficiently low densities of the perturbers the perturbed profile of a spectral line is Lorentzian and can be defined by two line parameters, the width and the shift of the main line. These quantities can be obtained in the impact limit of the general calculation of the autocorrelation function (Eq. (6)). Comparison of the unified profiles to the corresponding Lorentzian profiles may 


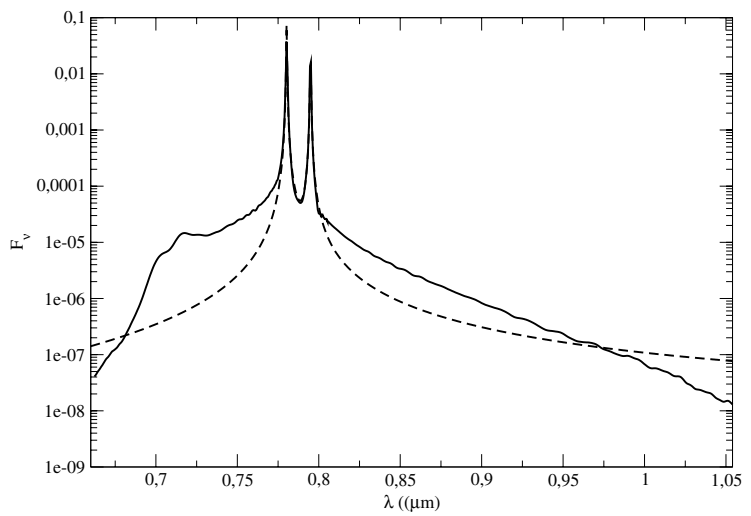

Fig. 11. $\mathrm{Rb}$ total unified line profile perturbed by $\mathrm{H}_{2}$ (full line), compared to the corresponding Lorentzian profile (dashed lines). The density of perturbers is $n_{\mathrm{H}_{2}}=10^{20} \mathrm{~cm}^{-3}$. The temperature is $1000 \mathrm{~K}$.

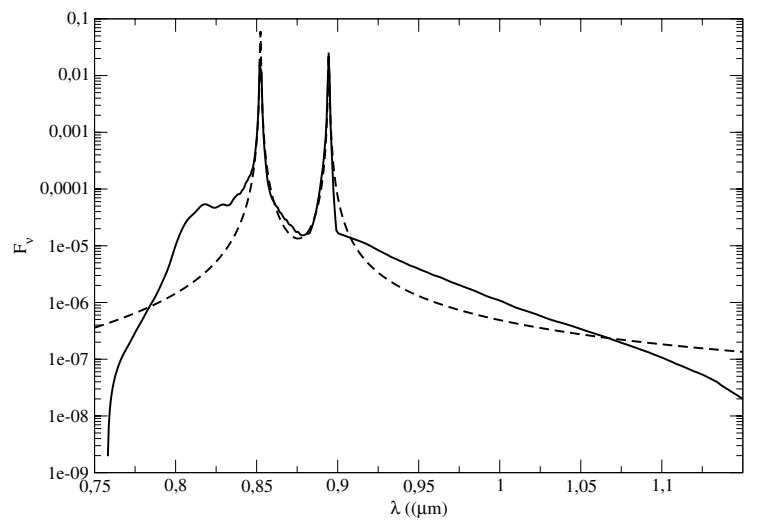

Fig. 12. Cs total unified line profile perturbed by $\mathrm{H}_{2}$ (full line), compared to the corresponding Lorentzian profile (dashed lines). The density of perturbers is $n_{\mathrm{H}_{2}}=10^{20} \mathrm{~cm}^{-3}$. The temperature is $1000 \mathrm{~K}$.

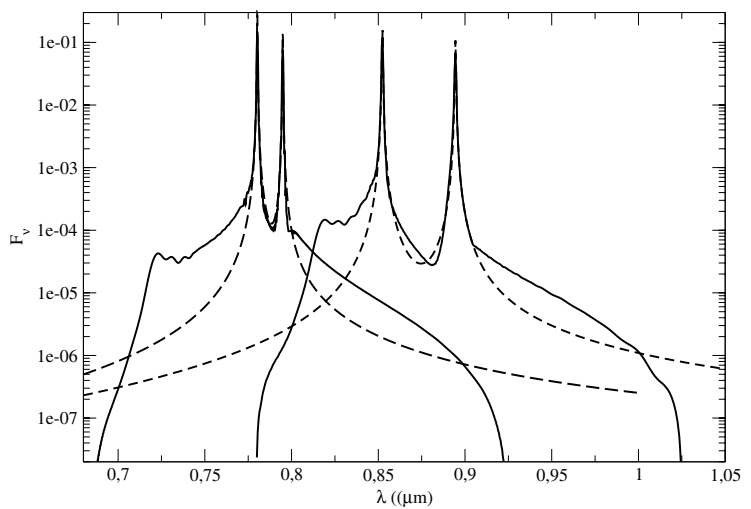

Fig. 13. $\mathrm{Rb}$ and $\mathrm{Cs}$ total unified line profiles perturbed by He (full line), compared to the corresponding Lorentzian profiles (dashed lines). The density of perturbers is $n_{\mathrm{He}}=10^{20} \mathrm{~cm}^{-3}$. The temperature is $1000 \mathrm{~K}$.

explain the missing opacity we noticed in Allard et al. (2003) (Figs. 11-13).

\section{Conclusion}

A unified semi-classical theory of line broadening has been used to determine the total profile for the center to the far wing

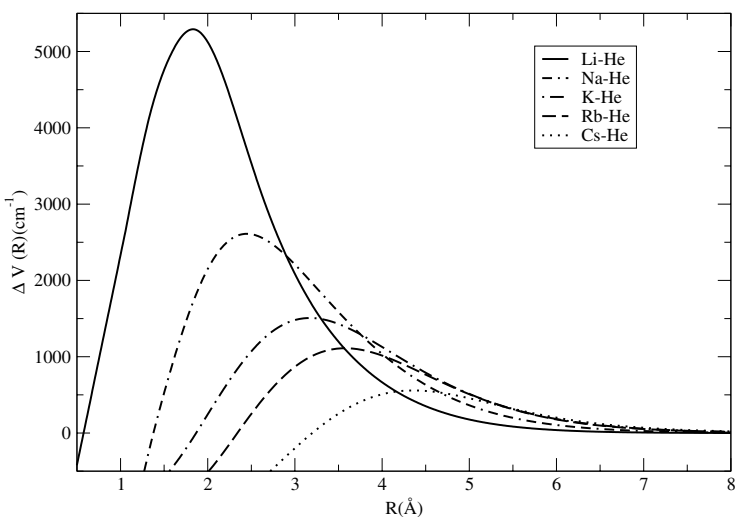

Fig. 14. $\Delta V(R)$ for the $\mathrm{B}^{2} \Sigma-\mathrm{X}^{2} \Sigma$ transition as a function of the internuclear distance $R$ for alkalis perturbed by He.

until $2000 \AA$ from the line center. The absorption profiles of the rubidium and the cesium resonance lines were calculated for physical conditions encountered in the atmospheres of brown dwarfs where the perturber density is high enough to get multiperturber effects on the profiles. An important characteristic of heavy alkalis is that the extremum of $\Delta V(R)$ is smaller and appears at a larger internuclear separation, up to $R=4.5 \AA$, as compared to the light alkalis (Fig. 14). This difference is important for the position and the amplitude of the satellites; the blue wing is then less extended and its strength is larger. These reasons may explain the missing opacity when using Lorentzian profiles instead of unified profiles, although the abundances of rubidium and cesium are small. The dependence of the position and strength of the line satellites on orientation angle requires us to know the potentials for more possible orientations of the $\mathrm{H}_{2}$ molecule and may lead to a significant improvement. The next step will be to include these new profiles in the model atmospheres and synthetic spectra of brown dwarfs.

\section{References}

Allard, F., Hauschildt, P. H., Alexander, D. R., Tamanai, A., \& Schweitzer, A. 2001, ApJ, 556,357

Allard, N. F. 1978, J. Phys. B., 11, 8, 1383

Allard, N. F., \& Kielkopf, J. F. 1982, Rev. Mod. Phys., 54, 1103

Allard, N. F., Koester, D., Feautrier, N., \& Spielfiedel, A. 1994, A\&A, 108, 417

Allard, N. F., Drira, I., Gerbaldi, M., Kielkopf, J. F., \& Spielfiedel, A. 1998, A\&A, 335, 1124

Allard, N. F., Royer, A., Kielkopf, J. F., \& Feautrier, N. 1999, Phys. Rev. A, 60, 1021 Allard, N. F., Allard, F., Hauschildt, P. H., Kielkopf, J. F., \& Machin, L. 2003, A\&A, 411, $\mathrm{L} 473$

Allard, N. F., Allard, F., \& Kielkopf, J. F. 2005, A\&A, 440, 1195

Anderson, P. W., \& Talman, J. D. 1956, Proc. Conf. Broadening of Spectral Lines. Bell Telephone System Technical Publications (NJ: Murray Hill), 3117, 29

Baranger, M. 1958a, Phys. Rev., 111, 481

Baranger, M. 1958b, Phys. Rev., 111, 494

Burrows, A., Marley, M. S., \& Sharp, C. M. 2000, ApJ, 531, 438

Burrows, A., \& Volobuyev, M. 2003, ApJ, 583, 985

Cohen, J. S., \& Schneider, B. 1974, J. Chem. Phys., 61, 3230

Gilbert, D. E., \& Ch'en, S. Y. 1969, Phys. Rev., 188, 40

Kielkopf, J. F., \& Allard, N. F. 1979, Phys. Rev. Lett., 43, 196

Mc Cartan, D. G., \& Hindmarsh, W. R. 1969, J. Phys. B: At Mol Phys., 2, 1396

Pascale, J. 1983, Phys. Rev. A, 28, 632

Pascale, J. 2003, Private communication

Rossi, F., \& Pascale, J. 1985, Phys. Rev. A, 32, 2657

Royer, A. 1978, Acta Phys. Pol. A, 54, 805

Royer, A. 1980, Phys. Rev. A, 22, 1625

Szudy, J., \& Baylis, W. 1975, J. Quant. Spectrosc. Radiat. Transfer, 15, 641

Szudy, J., \& Baylis, W. 1996, Phys. Rep., 266, 127 